\title{
Évaluation et gestion du risque radioactif suite au décès d'un patient ayant reçu une dose thérapeutique d'iode-131 dans le cadre d'un traitement en radiothérapie métabolique
}

\author{
C.F. DJOUMESSI ${ }^{1,2}$, C. BRAMOULLE ${ }^{2}$, C. PRUNIER $^{1}$, J.L. BAULIEU ${ }^{1}$, \\ J.C. BESNARD ${ }^{1}$
}

(Manuscrit reçu le 6 mars 2009, accepté le 12 mai 2009)

RÉSUMÉ Un patient traité à la méta-iodobenzylguanidine (mIBG) marquée à l'iode-131 pour un phéochromocytome malin est décédé trois heures après injection. Afin de minimiser les risques liés à la radioactivité, la décision de garder la dépouille pendant seize jours a été prise. Le présent travail présente les niveaux d'exposition des différentes personnes étant intervenues dans la prise en charge de la patiente depuis son hospitalisation jusqu'à son enterrement ainsi que les dispositions prises pour éviter toute contamination et dissémination radioactive.

ABSTRACT Radioactive's risk assessment and management following the death of a patient who received a therapeutic dose of iodine-131 as part of a treatment by radioimmunotherapy.

A patient treated with meta-iodobenzylguanidine (MIBG) marked with iodine-131 for malignant pheochromocytoma died three hours after injection. To minimize radioactive's risks, the decision to keep the remains for sixteen days was taken. This paper presents the levels of exposure of different persons involved in the care of the patient since his hospitalization until his funeral and the measures taken to prevent radioactive spread and contamination.

Keywords: Radioactive's risk / radio-immunotherapy

\section{Introduction}

Un patient traité à la mIBG et devant recevoir par injection une activité de $5484 \mathrm{MBq}$ d'iode-131 dans une seringue est admis en chambre d'hospitalisation de radiothérapie métabolique. Une heure et trente minutes après le début de l'injection, le patient est pris d'un malaise et se plaint de douleurs à la poitrine. Le traitement ayant été interrompu, il a été décidé de transférer le patient en

1 Service de médecine nucléaire, CHU de Tours, 2 boulevard Tonnelé, 37044 Tours Cedex 9, France.

2 Unité de Radiophysique, Clinique d'Oncologie et de Radiothérapie, CHRU de Tours, 2 boulevard Tonnelé, 37044 Tours Cedex 9, France. 
TABLEAU I

L'iode-131 et ses principales voies de désintégrations. Iodine-131 and its main modes of decays.

\begin{tabular}{|c|c|c|c|c|}
\hline \multirow[t]{2}{*}{ I-131 } & \multicolumn{2}{|c|}{ Rayonnements (keV) } & \multirow{2}{*}{$\frac{\text { Intensité (\%) }}{6}$} & \multirow{2}{*}{$\begin{array}{c}\text { Période physique (jrs) } \\
8,02\end{array}$} \\
\hline & $\gamma$ & 284 & & \\
\hline & & 365 & 82 & \\
\hline & & 637 & 7 & \\
\hline & $\beta-$ & 248 & 2 & \\
\hline & & 334 & 7 & \\
\hline & & 606 & 90 & \\
\hline
\end{tabular}

réanimation où il décèdera 3 heures après des suites d'un arrêt cardiaque. Face à l'activité déjà administrée au patient (estimée à $3656 \mathrm{MBq}$ ) et au souci d'assurer la radioprotection du personnel et de l'environnement, nous avons été amenés à prendre un certain nombre de mesures. Ces mesures ont permis d'avoir une estimation des niveaux d'exposition de tous les agents et d'enrayer tout risque de contamination radioactive depuis la chambre d'hospitalisation du patient jusqu'à son enterrement en passant par le service de réanimation et la chambre mortuaire.

\section{Matériel}

Le traitement des phéochromocytomes malins par radiothérapie métabolique se fait dans notre service de médecine nucléaire par injection de la métaiodobenzylguanidine (mIBG) marquée à l'iode-131(Giammarile, 2001). La métaiodobenzylguanidine est une molécule dérivée de la guanéthidine et proche structurellement de la noradrénaline qui se caractérise par une accumulation sélective dans les organes qui contiennent des catécholamines et des tumeurs qui dérivent de ces organes (phéochromocytomes; carcinomes médullaires thyroïdiens ; neuroblastomes et autres) (Gambini et Granier, 1988). La mIBG est marquée à l'iode-131 qui de par ses modes de désintégration (Tab. I) présente l'avantage d'offrir une possibilité d'imagerie en plus de son action thérapeutique.

Le suivi dosimétrique du personnel concerné inclut l'exposition au début du traitement jusqu'à l'enterrement. Il s'est déroulé de deux façons : le suivi du personnel travaillant habituellement en zone réglementée a été réalisé grâce au dosimètre opérationnel de service ${ }^{3}$; le suivi du personnel non recensé dans la base de dosimétrie opérationnelle a été réalisé par le biais de deux dosimètres personnels électroniques EPD Mk2 de Siemens distribués par la société APVL.

\footnotetext{
3 APVL Ingénierie - dosimètre personnel électronique EPD MK2 Siemens
} 
Le détecteur EPD Mk2 est à lecture directe grâce à la diode PIN intégrée et le détecteur APVL est lu sur une console informatique permettant ainsi d'avoir accès à l'équivalent de dose individuel $H p(10)$ (ICRP, 1991 ; ICRU, 1985). Ces détecteurs sont appropriés pour des mesures aux expositions X, gamma et bêta, ceci dans les gammes d'énergie suivantes :

- bêta (énergie moyenne) : $250 \mathrm{keV}$ à $1,5 \mathrm{MeV}$;

- rayons $\mathrm{X}$ et gamma : $15 \mathrm{keV}$ à $10 \mathrm{MeV}$.

Ces dosimètres sont opérationnels pour les réponses en $H p(10)$ sur une plage allant de $1 \mu \mathrm{Sv}$ à $16 \mathrm{~Sv}$.

Un détecteur de type PRM 610 a été utilisé pour vérifier toute éventualité de contamination surfacique. D'autre part, nous avons utilisé un dosimètre/débitmètre de type babyline 81 à réponse linéaire muni d'un capuchon d'équilibre électronique et ayant les caractéristiques suivantes :

- gamme de débit de dose : $10 \mu \mathrm{Gy} / \mathrm{h}$ à $1000 \mathrm{mGy} / \mathrm{h}$;

- dose absorbée : $10 \mu \mathrm{Gy}$ à $1000 \mathrm{mGy}$;

- réponse uniforme de $8 \mathrm{keV}$ à $2 \mathrm{MeV}$ pour les photons et les électrons.

\section{Méthode}

La particularité de cet évènement est qu'il a fait appel aux compétences de plusieurs services autres que celui de médecine nucléaire. Les 31 personnes suivies sont les suivantes :

- 6 médecins et étudiants en médecine (médecine nucléaire et réanimation) ;

- 1 radiophysicien (médecine nucléaire) ;

- 1 manipulateur d'électroradiologie médicale ;

- 4 infirmières (médecine nucléaire et réanimation);

- 3 aide-soignantes hospitalières (réanimation);

- 4 brancardiers et ambulanciers ;

- 2 agents de la conciergerie ;

- 8 agents de la morgue ;

- 2 agents des pompes funèbres.

Sur le plan de la classification du personnel, les agents cités ci-dessus sont regroupés comme suit :

- catégorie $A$ : une infirmière et un manipulateur ;

- catégorie B : 2 médecins et le radiophysicien ;

- public : tout le reste.

Le suivi dosimétrique du personnel qui est présenté dans ce travail commence dès le début du malaise du patient. Le personnel de catégories A et B étant affecté au 
système de dosimétrie opérationnel de l'hôpital, leur dose reçue a été lue à partir de la base d'enregistrement. Ne disposant que de 2 dosimètres EPD, le suivi des agents appartenant à la catégorie « public » s'est fait par relevés successifs de dose individuelle après passage auprès du patient.

Le détecteur de type PRM 610 a été utilisé pour contrôler la non contamination des mains, des pieds et de matériel utilisé après toute intervention dans la chambre du patient. Des surchaussures, gants à usage unique et surblouses ont été mis à la disposition du personnel afin d'éviter toute contamination

La dépouille a été placée dans une housse doublée pour limiter tout éventuel écoulement et entreposée dans le casier le plus à l'extrémité de la chambre mortuaire. Des affiches de signalement de risque radioactif et de conduite à tenir ont été placées autour de la pièce en même temps que les occupants des services voisins ont été informés des risques radioactifs.

Dans le but de limiter l'exposition des membres de la famille et autre personne appelés à être présent pendant l'inhumation, le séjour de la dépouille dans notre service mortuaire a été ajusté à la période physique de décroissance de l'iode-131. De plus, le cercueil a été doublé d'une couche de trois millimètres de plomb représentant une demi-atténuation pour le débit de dose ambiant.

\section{Résultats}

\subsection{Chambre d'hospitalisation}

Pour le personnel intervenant dans la chambre d'hospitalisation entre le début du malaise du patient et son transport au service de réanimation, la dose efficace a été relevée. Le niveau d'exposition (voir Fig. 1) est dû à l'urgence de la situation ayant nécessité une intervention de tout le personnel présent.

La forte exposition du personnel peut en partie être expliquée par le souci de limiter au maximum la dissémination de la contamination radioactive. Les premiers soins au patient ayant nécessité une proximité avec ce dernier, cela explique le niveau d'exposition du médecin $(152 \mu \mathrm{Sv})$. Les niveaux d'exposition de l'infirmière et du manipulateur en électroradiologie médicale respectivement de 149 et $118 \mu \mathrm{Sv}$ sont une illustration de leur rôle important dans la prise en charge de la patiente. Le pic maximal de débit de dose relevé dans la chambre d'hospitalisation était de $1228 \mu \mathrm{Sv} / \mathrm{h}$. 


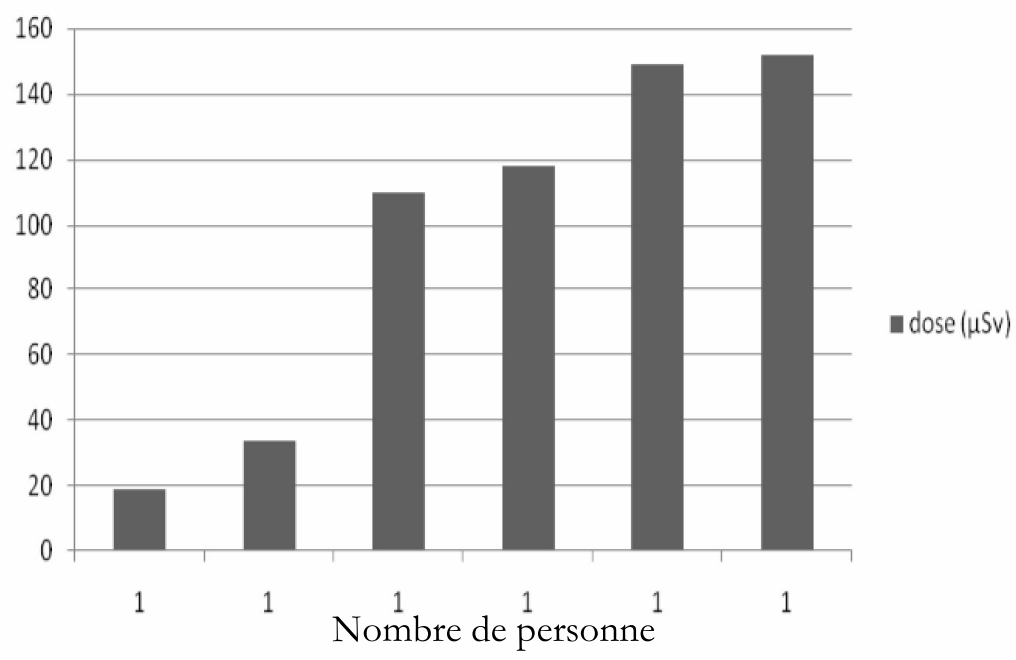

Figure 1 - Équivalent de dose individuel Hp(10) reçu par le personnel dans la chambre d'hospitalisation (dosimétrie poitrine).

Individual dose equivalent $\mathrm{Hp}(10)$ received by staff who have intervened in the hospital room (chest dosimetry).

\subsection{Service de réanimation}

Le patient a subi à cette étape un massage cardiaque, ce qui a nécessité l'intervention et la présence d'un nombre important de personnes dans la pièce pendant une durée estimée à quinze minutes. Le patient y a séjourné trois heures avant son décès. Au moment du transfert de la dépouille en chambre mortuaire, on comptait six heures de présence de radioactivité dans le service. La figure 2 présente l'exposition de tous les agents du service de réanimation ayant pris part aux soins.

L'étape du massage cardiaque a contribué pour $25 \mu \mathrm{Sv}$ dans la dosimétrie du personnel. La préparation et les soins au patient pré et post-mortem expliquent les niveaux d'exposition élevés au cours de cette phase. L'aide soignante et l'infirmière en charge de cette préparation ont enregistré les doses les plus élevées respectivement 140 et $96 \mu \mathrm{Sv}$. Ces valeurs ont été enregistrées sur une demijournée de travail. Le débit de dose maximal relevé dans la chambre du patient était de $925 \mu \mathrm{S} / \mathrm{h}$. 


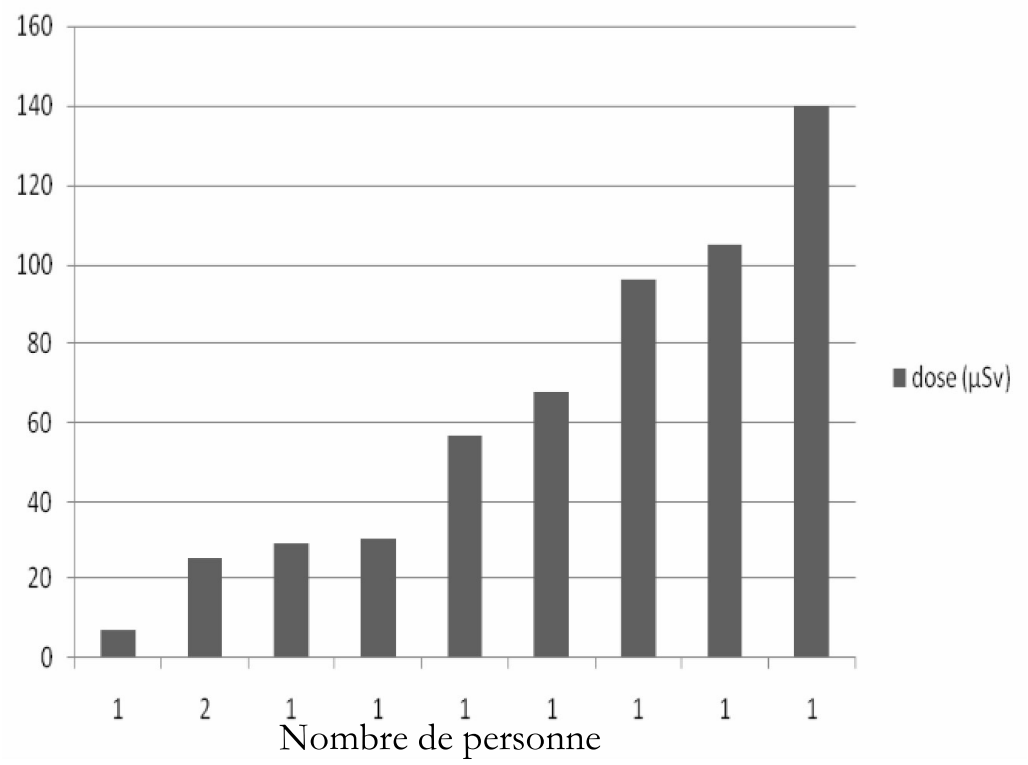

Figure 2-Équivalent de dose individuel Hp(10) reçu par le personnel au service de réanimation (dosimétrie poitrine).

Individual dose equivalent $H p(10)$ received by staff who have intervened in the intensive care unit (chest dosimetry).

\subsection{Transport}

Nous entendons ici par transport, les différentes étapes au cours desquelles la dépouille a dû être déplacée du service de réanimation (dans lequel est survenu le décès) au cimetière en passant par la chambre mortuaire et la morgue. Des dosimètres opérationnels ont été remis au personnel des pompes funèbres lors du transport de la dépouille afin d'estimer leur exposition. La figure 3 inclut les 2 agents des pompes funèbres ainsi que les ambulanciers et brancardiers de notre établissement. Le débit de dose maximal relevé pendant ces différentes étapes a été de $42 \mu \mathrm{Sv} / \mathrm{h}$.

\subsection{La morgue}

Nous présentons ici le niveau d'exposition du personnel du service mortuaire pendant toute la durée du séjour de la dépouille à la morgue. La rotation des équipes a permis un étalement de l'exposition du personnel. L'agent le plus exposé 


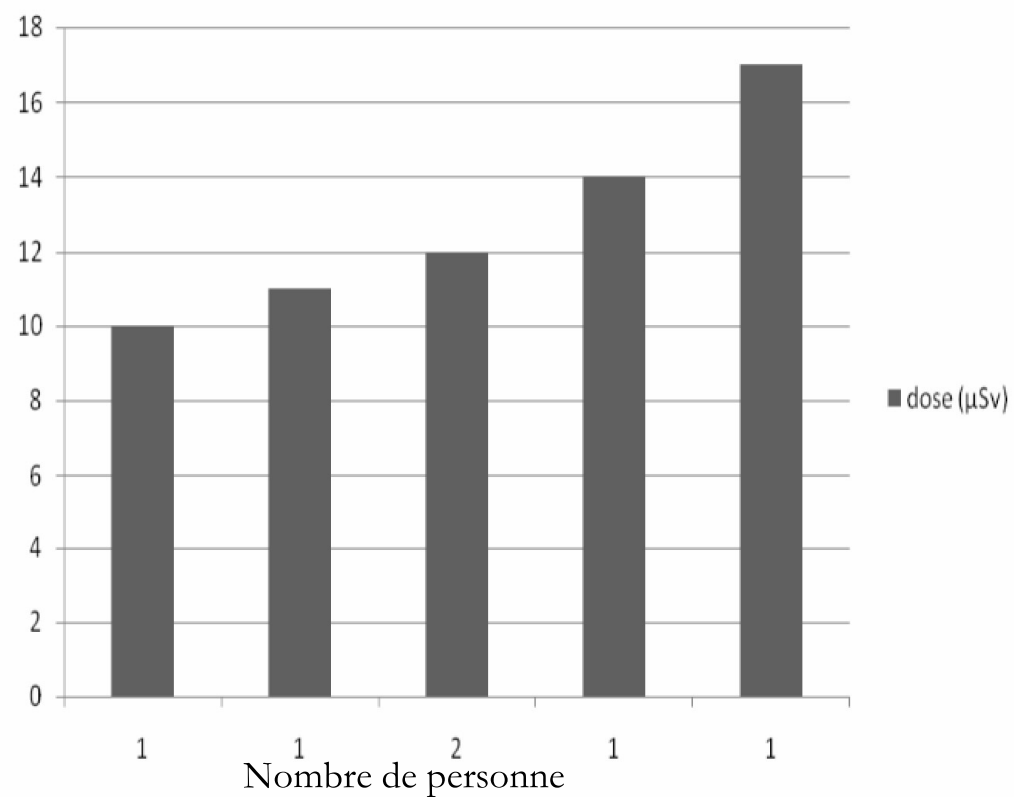

Figure 3 - Équivalent de dose individuel Hp(10) reçu par le personnel lors du transport de la dépouille jusqu'à son enterrement (dosimétrie poitrine).

Individual dose equivalent $\mathrm{Hp}(10)$ received by the personnel involved in transporting the body to its burial (chest dosimetry).

( $94 \mu \mathrm{Sv}$ ) est celui qui a procédé à la préparation de la dépouille à l'arrivée dans le service. La figure 4 présente les niveaux d'exposition des agents concernés.

Ces niveaux de dose représentent l'exposition enregistrée au cours des 16 jours que la dépouille a passé dans le service mortuaire.

\subsection{Mesure de débit de dose ambiant}

Nous avons procédé à l'aide de la babyline à des mesures de débit de dose ambiant auprès de la dépouille, autour du casier contenant la dépouille et autour du cercueil dans lequel elle a été placée.

Le tableau II donne les mesures de débit de dose ambiant autour de la dépouille immédiatement après le décès, 12 heures après et au cinquième jour. Le tableau III présente le débit de dose ambiant à J1, J2, J5 autour du casier dans lequel a été 


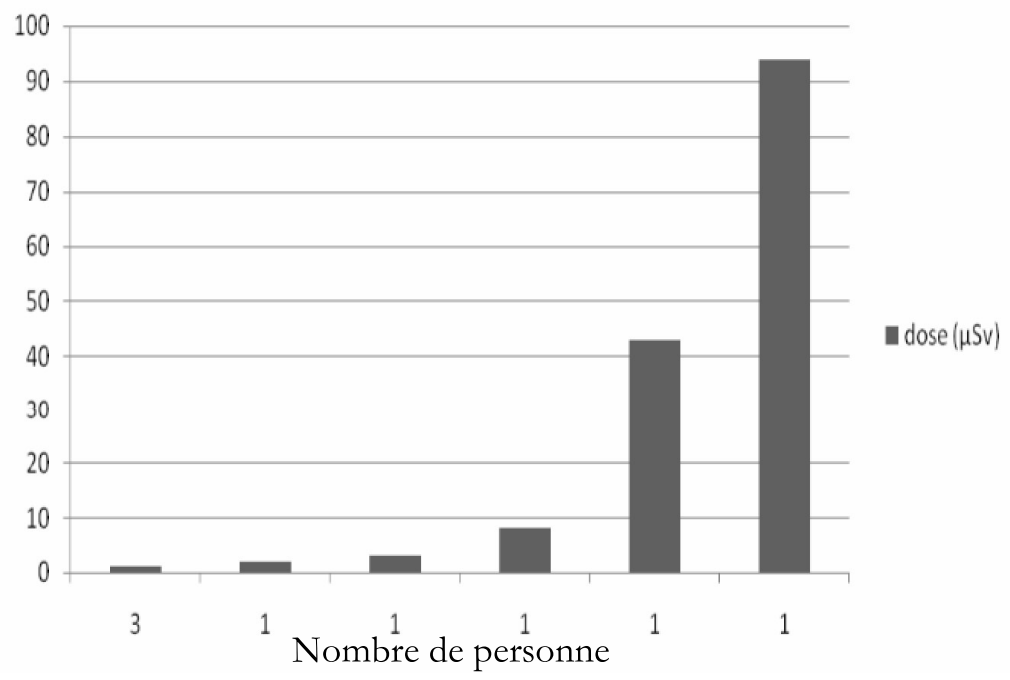

Figure 4-Équivalent de dose individuel Hp(10) reçu par le personnel du service mortuaire (dosimétrie poitrine).

Individual dose equivalent $\mathrm{Hp}(10)$ received by the mortuary staff (chest dosimetry).

\section{TABLEAU II}

Débit de dose ambiant en $\mu \mathrm{Sv} / \mathrm{h}$ au contact et à $100 \mathrm{~cm}$ de la dépouille à $\mathrm{J0}, \mathrm{J} 1$ et $\mathrm{J5}$. Ambient dose rate in $\mu \mathrm{Sv} / \mathrm{h}$ at contact and at $100 \mathrm{~cm}$ of the mortal on day 0 , day 1 and day 5 .

\begin{tabular}{cccc}
\hline & J0 & J1 & J5 \\
\hline Contact & 1600 & 1550 & 880 \\
$100 \mathrm{~cm}$ & 95 & 70 & 44 \\
\hline
\end{tabular}

TABLEAU III

Débit de dose ambiant en $\mu \mathrm{Sv} / \mathrm{h}$ au contact et à $100 \mathrm{~cm}$ du casier de la morgue à $\mathrm{J1}, \mathrm{J} 2$ et $\mathrm{J5}$. Ambient dose rate in $\mu \mathrm{Sv} / \mathrm{h}$ at contact and at $100 \mathrm{~cm}$ of the morgue's trap on day 1 , day 2 and day 5 .

\begin{tabular}{cccc}
\hline & J1 & J2 & J5 \\
\hline Contact & 30 & 28 & 22 \\
$100 \mathrm{~cm}$ & 10 & 8 & 5 \\
\hline
\end{tabular}

conservée la dépouille. Les tableaux II et III montrent une décroissance du débit de dose suivant la période de l'iode-131.

Les mesures effectuées autour du cercueil ont montré un débit de dose ambiant de $22 \mu \mathrm{Sv} / \mathrm{h}$ à un mètre. Ces dernières nous ont permis de donner des 
recommandations plus strictes au personnel des pompes funèbres ainsi qu'aux membres de la famille de la défunte par rapport à la conduite à tenir pendant les obsèques.

\section{Conclusion}

La difficulté majeure dans la gestion d'un tel évènement est qu'il implique des agents non habitués à travailler en présence des rayonnements ionisants. Celle-ci peut être surmontée par une communication efficace, adaptée à l'urgence et à la singularité de la situation. Cette difficulté est d'autant plus accentuée qu'un tel évènement inclut la famille du défunt.

Dans le cas présent, la gestion a été organisée sous forme tripartite :

- le corps médical : il s'est chargé de l'annonce du décès à la famille du défunt en leur présentant la particularité de la situation au vu du risque radioactif ;

- l'administration hospitalière : par l'outil de communication mis en place et l'accompagnement de proximité accordée à la famille, elle a donné une bonne lisibilité à la gestion de l'évènement. Par ailleurs, elle a su rassurer et mobiliser le personnel autour de la situation ;

- le service de radioprotection et l'unité de radiophysique ont apporté leur expertise tant au niveau de la famille, de la morgue et des autres services afin d'apporter les informations nécessaires liées aux risques dus à l'exposition aux rayonnements ionisants, au niveau d'exposition du personnel et à la gestion du risque radioactif.

En interne, nous disposons d'une procédure encadrant la sortie prématurée de tout patient ayant reçu une dose thérapeutique en radiothérapie métabolique. Cette procédure est bien connue des agents intervenant en thérapie métabolique.

Le système de dosimétrie opérationnelle en service dans notre établissement nous a permis d'évaluer l'exposition des agents. Les données dosimétriques du personnel ont été communiquées à la médecine du travail pour le suivi des agents.

Notre établissement, par le biais de son service compétent en radioprotection, s'est entouré de l'expertise de l'ASN et de l'IRSN pour cette situation.

Pour un patient ayant reçu un radiopharmaceutique à visée thérapeutique, sa sortie d'hospitalisation dans notre service est conditionnée par un débit de dose ambiant inférieur ou égal à $25 \mu \mathrm{Sv} / \mathrm{h}$. Le débit de $22 \mu \mathrm{Sv} / \mathrm{h}$ mesuré à un mètre du cercueil nous a permis de remettre la dépouille à la famille ; cette dernière après explication de nos responsables a consenti à annuler à la crémation voulue par le défunt. 
Le constat qui se dégage au terme de ce travail est qu'il est nécessaire que des réflexions au niveau national soient menées afin d'encadrer la gestion des évènements tels que le décès d'un patient ayant reçu une dose thérapeutique (médecine nucléaire, curiethérapie de prostate, etc.).

Remerciements. Les auteurs remercient la direction hospitalière, les équipes des services de médecine nucléaire in vivo, de réanimation 3 et de l'institut médicolégal du CHRU de Tours.

\section{RÉFÉRENCES}

Gambini Dj., Granier R. (1988) Manuel pratique de médecine nucléaire. EM Inter, Paris

Giammarile F. (2001) Le traitement du neuroblastome par radiothérapie métabolique, Imagerie fonctionnelle et métabolique 25(4).

ICRP Publication 60 (1991) 1990 Recommendations of the International Commission on Radiation Protection, Ann. ICRP 21(1-3)

ICRU (1985) Report 39, Determination of Dose Equivalents Resulting from external Radiation Sources. 